\title{
A Modified Wire-Loop Snare Technique to Retrieve Inferior Vena Cava Filters with Severe Tilt and Embedded Hooks
}

\author{
Yuewei Wang ${ }^{1,}$, , Haofu Wang ${ }^{1}$, Hai Xin ${ }^{1}$ and Jun $\mathrm{Li}^{1}$ \\ ${ }^{1}$ Department of Vascular Surgery, Affiliated Hospital of Qingdao University, Qingdao, China \\ "Corresponding author: Department of Vascular Surgery, Affiliated Hospital of Qingdao University, 266003, Qingdao, China. Email: wangyw791128@163.com
}

Received 2018 September 19; Revised 2019 May 20; Accepted 2019 June 01

\begin{abstract}
Background: Severe tilt with embedded hook is a common obstacle to successful retrieval of the retrievable inferior vena cava (IVC) filter. One reason leading to retrieval failure is the guide wire and catheter that could not be guided between the IVC wall and filter neck.

Objectives: The aim of this study is to investigate the feasibility of a modified wire-loop technique for retrieval of inferior vena cava filters with severe tilt and embedded hooks.

Patients and Methods: Between September 2013 and November 2017, 156 patients underwent IVC filter implantation including drum-type filter (OptEase) and conical filter (Celect). Preoperative CT angiography or intraoperative angiogram revealed that three drum-type filters and four conical filters tilted severely and could not be retrieved using a conventional snare technique. We performed a modified wire-loop snare technique. The end of 5F pigtail catheter was cut off and remade to be fishhook-like. The pigtail catheter and 0.035-inch-long soft guide wire were introduced into the inferior vena cava (IVCF) through long sheath. A $5 \mathrm{~F}$ vertebral catheter and a snare were advanced into the IVCF simultaneously. The pigtail catheter directed the guide wire to pass through the interstice of the cava wall and filter neck. The leading end of the wire was snared by $5 \mathrm{~F}$ vertebral catheter and the snare to form a loop. Then the loop snared the hook. If there was no interstice between the filter neck and cava wall, the homemade catheter would then direct the guide wire to pass through the interstice of separate filter struts. The conventional technique was performed or the long sheath advanced over the wire-loop to retrieve the filter directly.

Results: Four conical filters were retrieved by snaring the hook directly. The filter hook of drum-type filter was stretched away from the cava wall in two cases, and then, the conventional snare technique was performed successfully. The long sheath was advanced over the wire-loop to retrieve the filter directly in one case.

Conclusion: The modified wire-loop snare technique is simple and effective and can be used to retrieve both conical and drum-type filters with severe tilt and embedded hooks.
\end{abstract}

Keywords: Inferior Vena Cava Filter, Severe Tilt, Embedded Hook, Pigtail Catheter, Wire-Loop

\section{Background}

Pulmonary embolism caused by deep venous thrombosis (DVT) is a significant cause of death. An inferior vena cava (IVC) filter can reduce the incidence of fatal pulmonary embolism. However, a variety of complications related to the IVC filter may occur, including migration, thrombosis and IVC obstruction with long-term implantation $(1,2)$. Retrievable filters could be more beneficial to patients because they result in fewer complications when compared with permanent filters (3).

However, severe tilt and embedded hooks are common obstacles to successful recovery of IVC filter. Severe tilt means that the hook of the filter attaches to the vena cava wall. The hook of the filter could be embedded by hyperplastic intima and thrombus after the hook attaches to ves- sel wall. The main reason resulting in retrieval failure is that the catheter and the wire cannot pass through the interstice of the filter neck and cava wall (4). Some advanced techniques used for this difficult retrieval, such as the fallback and hangman techniques, have been described $(4,5)$. However, the retrieval success rate of these techniques did not achieve $100 \%$ with no complications. Moreover, these techniques were used for retrieval of conical filters, such as Celect and Gün-ther Tulip, and a larger sheath (14F, or even $18 \mathrm{~F}$ ) was used in the retrieval process.

\section{Objectives}

Herein, we describe a modified wire-loop snare technique with homemade fishhook-like pigtail catheter to re- 
trieve two types of filters (OptEase; Cordis, Fremont, California, and Celect; Cook Medical, Bloomington, Indiana). The purpose of this study is to investigate the feasibility of the modified wire-loop technique for retrieval of inferior vena cava filters with severe tilt and embedded hooks.

\section{Patients and Methods}

\subsection{Patients}

Between September 2013 and November 2017, 156 patients in our department underwent IVC filter implantation to prevent fatal PE before catheter-directed thrombolysis, percutaneous transluminal angioplasty of the iliac and femoral vein, and resection of pelvic mass. We preferred to choose retrievable filters and two types of filters were available in our hospital including drum-type filter (OptEase) and conical filter (Celect). The time to retrieve drum-type filter and conical filter was within 14 days and within 3 months after implantation, respectively. So we evaluated the condition of the patient before filter implantation. If the filter could be retrieved within 14 days, we preferred to choose OptEase. If the filter could not be retrieved within 14 days, we preferred to choose Celect. 121 drum-type filters and 35 conical filters were implanted. After filter implantation, the patients needed long-term anticoagulant therapy and were given warfarin or rivaroxaban according to their choice. Four conical filters and three drum-type filters with severe tilt and embedded hooks could not be retrieved by the conventional snare technique (Table 1). Preoperative CT angiography or intraoperative angiogram of different projections including front, oblique, lateral revealed that the tilt hook was in the vessel wall and not in the lumen.

\subsection{Methods}

We performed a modified wire-loop snare technique to retrieve these four conical and three drum-type filters. All filters were planned to retrieve after a certain time. The duration of implantation ranged from 12 to 16 days for drumtype filters and 35 to 60 days for conical filters, as shown in Table 1.

For conical retrievable filters, preoperative CT angiography revealed tilt filter with embedded hook (Figure 1A). The venogram confirmed that the filter tilted severely (Figure $1 \mathrm{~B}$ ). An 11F long sheath (Cook Medical, Bloomington, IN) was placed via the right side internal jugular vein approach. The conventional snare technique failed to snare the hook. The end of $5 \mathrm{~F}$ pigtail catheter (Cordis, Fremont, CA) was cut off and remade to be fishhook-like (Figure 1C). The 0.035-inch-long soft guide wire (Terumo Corporation, Shibuya-ku, Tokyo) and homemade pigtail catheter were introduced into the IVC through long sheath. A snare(Lepu
Medical, Beijing) and 5F vertebral catheter (Cordis, Fremont, CA) were placed at the proximal IVC. The homemade pigtail catheter was placed at the interstice of cava wall and filter neck. The guide wire was passed through the interstice of cava wall and filter neck and advanced back. (Figure1D) The leading end of the guide wire was snared by the snare and vertebral catheter (Figure $1 \mathrm{E}$ ). The guide wire was withdrawn slowly to form a wire-loop and confirm that the hook was snared (Figure $1 \mathrm{~F}$ ). Then, the leading end of the guide wire was withdrawn into the sheath (Figure $1 G$ ). The trailing end and the leading end of the guide wire were stretched cranially (Figure $1 \mathrm{H}$ ). The sheath advanced over the wire-loop to collapse the filter (Figure 1I). Completion angiogram showed patent IVC with no rupture (Figure 1J).

For drum-type filters, the venogram showed that the filter tilted with the hook embedded in the cava wall, and there was no interstice between cava wall and filter hook (Figure 2A). The homemade pigtail catheter (Cordis) guided the 0.035 -inch-long soft guide wire (Terumo Corporation) to pass through the interstice of the filter struts and advance back into the snare (Lepu Medical; Figure 2B). The leading end of the guide wire was snared and formed a wire-loop to stretch the filter hook away from the cava wall (Figure $2 \mathrm{C}$ ). The hook was snared by the snare and $5 \mathrm{~F}$ vertebral catheter (Cordis; Figure 2D). The 12F long sheath (St. Jude Medical, Saint Paul, Minnesota) was advanced over the catheter and the snare to collapse the filter after withdrawing the pigtail catheter (Figure 2E). An organized thrombus and hyperplastic intima adhered to the filter and remained embedded in the filter hook (Figure 2F). If the wire-loop was unable to stretch the filter hook away from the IVC wall, the long sheath was advanced over the wire-loop to retrieve the filter directly. The homemade pigtail catheter guided the 0.035-inch-long soft guide wire (Terumo Corporation) to pass through the interstice of the filter struts and advance back into the snare (Lepu Medical; Figure $2 \mathrm{G}$ ). The leading end of the guide wire was snared by the snare and vertebral catheter (Cordis; Figure $2 \mathrm{H}$ ). The guide wire was withdrawn slowly to form a wire-loop and confirm that the hook was snared (Figure 2I). Then, the leading end of the guide wire was withdrawn into the sheath (Figure 2J). The trailing end and the leading end of the guide wire were stretched simultaneously and the sheath(St. Jude Medical) was advanced over the wire-loop (Figure $2 \mathrm{~K}$ ). The filter was retrieved directly by the wire-loop, and organized thrombus and hyperplastic intima adhered to the filter (Figure 2L).

The technique was approved by the Ethics Committee of the Affiliated Hospital of Qingdao University. The patients agreed and signed the informed consent. 

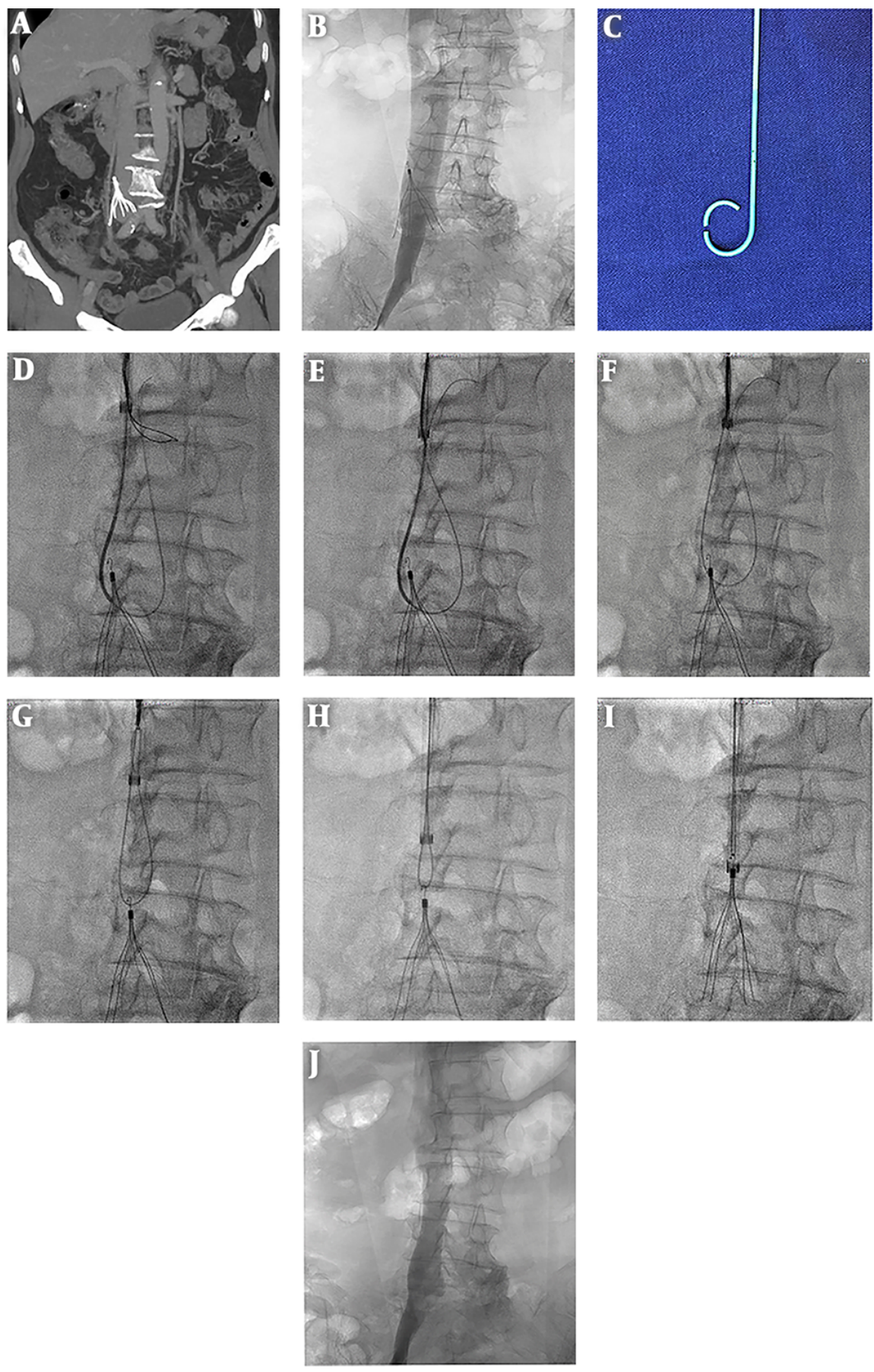

Figure 1. A, Preoperative CT angiography revealed tilt filter with embedded hook. B, The venogram confirmed that the filter tilted severely and there was an interstice between the filter neck and the inferior vena cava (IVC) wall. C, A 5F pigtail catheter was remade to be fishhook-like by cutting off the end. D, The soft guide wire was directed through the interstice of the filter neck and the IVC wall and advanced back into the snare. E, The leading end of the guide wire was snared by the snare and vertebral catheter. F, The guide wire was withdrawn slowly to confirm that the hook was snared. G, The leading end of the guide wire was withdrawn into the sheath. H, Cranially directed tension was applied to the trailing end and the leading end of the guide wire. I, The sheath was advanced over the wire-loop to collapse the filter. J, Completion angiogram showed patent IVC. 


\begin{tabular}{|c|c|c|c|c|c|c|c|}
\hline Case & Gender & Age & Diagnosis & Treatment & Type of filter & DOI & OT, min \\
\hline $\mathbf{1}$ & Male & $65 y$ & IFVE, PE, Cockett syndrome & PTA, CDT, iliac stent & OptEase & $12 \mathrm{~d}$ & 20 \\
\hline 2 & Male & $63 y$ & IFVE, PE & PTA, CDT & OptEase & $14 \mathrm{~d}$ & 25 \\
\hline 3 & Female & $58 y$ & IFVE, PE & PTA, CDT & OptEase & $16 \mathrm{~d}$ & 17 \\
\hline 4 & Male & $70 y$ & IVC and iliac thrombosis & CDT & Celect & $60 d$ & 25 \\
\hline 5 & Male & $75 y$ & IFVE & PTA, CDT & Celect & $56 \mathrm{~d}$ & 25 \\
\hline 6 & Female & $64 y$ & Femoral vein thrombosis & Resection of pelvic mass & Celect & $35 \mathrm{~d}$ & 30 \\
\hline 7 & Male & $67 y$ & IFVE & PTA, CDT & Celect & $60 d$ & 25 \\
\hline
\end{tabular}

Abbreviations: CDT, catheter-directed thrombolysis; d, days; DOI, duration of implantation; IFVE, iliac and femoral vein thrombosis; IVC, inferior vena cava; OT, operation time; PE, pulmonary embolism; PTA, percutaneous transluminal angioplasty

\section{Results}

The seven patients included two women and five men (age range, 58 - 75 years; mean age, 66 years). Four conical filters (Celect) were retrieved directly using the wireloop snare technique to snare the hook. The hooks of three drum-type filters (OptEase) could not be snared by the wire-loop. The filter hook was stretched away from the cava wall using the wire-loop snare technique in two cases, and the patients underwent standard snare technique successfully. The hook could not be stretched away from the cava wall in one case, so the long sheath was advanced over the wire-loop to retrieve the filter directly. We performed a modified wire-loop snare technique to retrieve all the filters in these seven patients with no associated complications. Intraoperative completion angiogram of every patient after filter retrieval showed patent vena cava and no sign of bleeding. The patients had no symptom of hematoma or arteriovenous fistula of puncture site, retroperitoneal hematoma, pulmonary embolism, and infection perioperatively. The operation time was from 17 to 30 minutes (Table 1). All the patients did not undergo the new filter insertion after retrieval. All the patients were discharged two days after operation and remained in good condition at follow-up.

\section{Discussion}

About 30\% of filters tilted before retrieval in our department. If severe tilt and embedded hook did not occur, the filters could be retrieved with routine snare technique. Severe tilt and embedded hooks are common obstacles of retrieving IVC filters successfully. The conventional snare technique is unable to snare the filter hook. In the present report, we described a modified wire-loop snare technique to snare the filter hook or the strut apex. This technique allows for direct retrieval of the filter or stretching the filter hook away from the cava wall to allow the conventional snare technique to be performed successfully.
One factor that contributed to retrieval failure of retrievable IVC filters with severe tilt and embedded hooks was that the catheter and the wire were unable to pass through the interstice of the cava wall and filter neck. Many advanced techniques for this difficult retrieval have been described. Al-Hakim et al. reported a hangman technique that created a wire-loop between cava wall and filter neck to release the embedded filter hook using a $14 \mathrm{~F}$ sheath; the retrieval rate was $81.9 \%$ (4). Foley et al. reported a fallback technique for filter retrieval by creating a wireloop between the legs of the filter using an $18 \mathrm{~F}$ sheath; the success rate was 96\% (5). Van Ha et al. introduced the balloon displacement technique by inflating a balloon between the apex of the filter and its embedded struts to detach the embedded struts from the cava wall (6). Others reported that the forceps technique was effective and safe for the removal of filters with severe tilt and embedded hook $(7,8)$. The dual access snare-over-wire method was shown to be feasible in case of difficult removal (9). However, it was not possible for the retrieval success rate to achieve $100 \%$ without complications. Moreover, these techniques were used for the retrieval of conical filters, and a larger sheath $(14 \mathrm{~F}$, or even $18 \mathrm{~F})$ was used.

In a previous report, we retrieved a conical filter (Celect) with severe tilt successfully using this wire-loop technique (10). In this report, we performed the wireloop snare technique to retrieve two types of retrievable filters (Celect and OptEase). This modified technique is effective, simple and uses standard equipment. It can improve the retrieval success rate with minor injury. First, some catheters, such as the RIM, may be suitable for directing the guide wire to form a loop. These catheters are not available in our institute. Therefore, we modified the wireloop snare technique by cutting off the end of the pigtail catheter based on the filter type and embedded hook. This fishhook-like catheter was able to direct the guide wire to easily pass through the interstice of the cava wall and filter neck. Second, for conical filters, snaring the leading end 

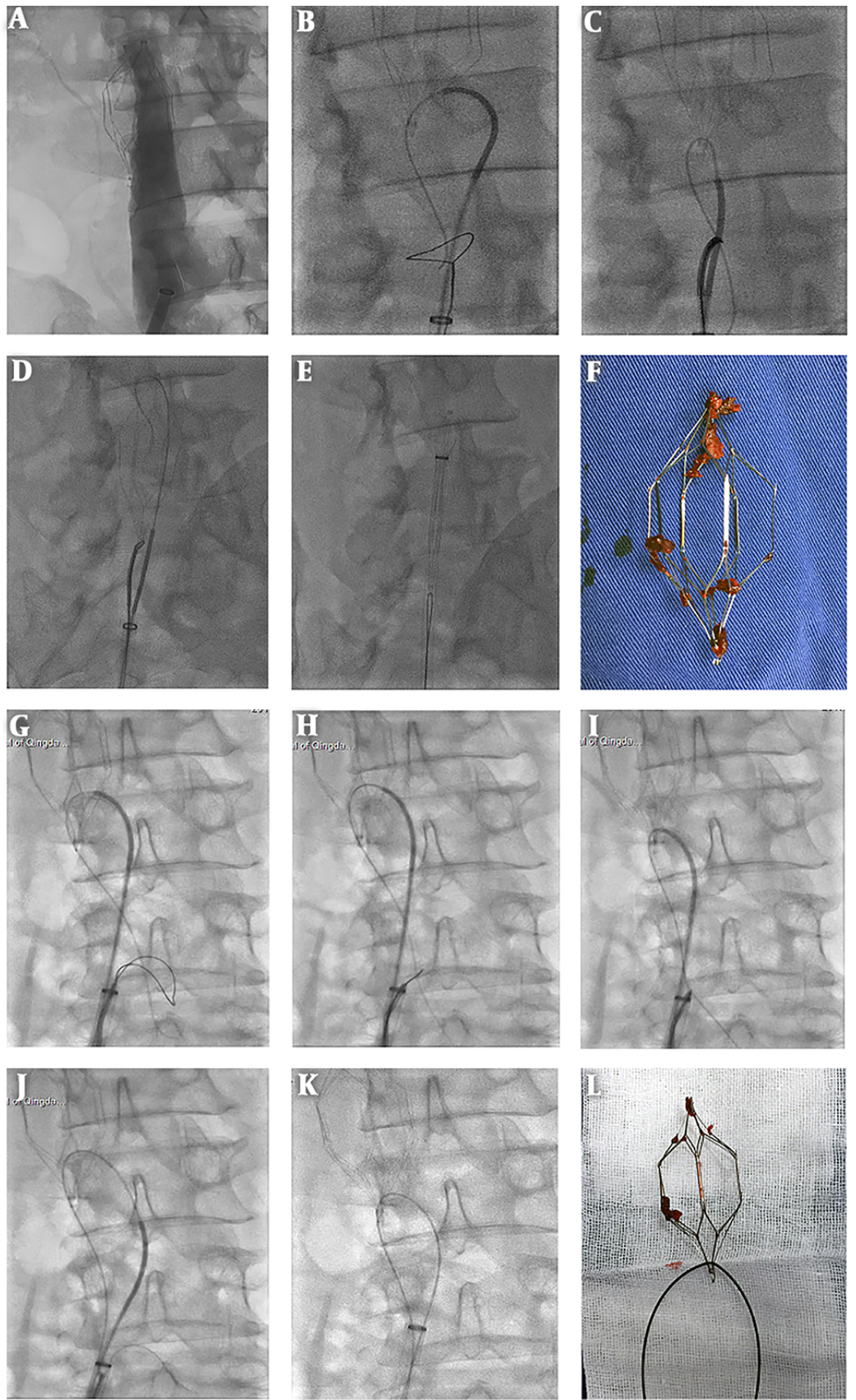

Figure 2. A, The venogram confirmed that the filter hook was embedded in the cava wall, and there was no interstice between the filter hook and the cava wall. B, The homemade pigtail catheter guided the 0.035-inch-long soft guide wire to pass through the interstice of the filter struts and advance back into the snare. C, The leading end of the guide wire was snared to form a loop and stretched the filter hook away from the cava wall. D, The hook was snared by the snare and $5 \mathrm{~F}$ vertebral catheter. E, The long sheath was advanced over the catheter and the snare to collapse the filter. F, An organized thrombus and hyperplastic intima adhered to the filter. G, The homemade pigtail catheter guided the 0.035 -inch-long soft guide wire to pass through the interstice of the filter struts and advance back into the snare. $H$, The leading end of the guide wire was snared by the snare and vertebral catheter. I, The guide wire was withdrawn slowly to form a wire-loop and confirm that the hook was snared. J, The leading end of the guide wire was withdrawn into the sheath. K, The trailing end and the leading end of the guide wire were stretched simultaneously and the sheath was advanced over the wire-loop. $\mathrm{L}$, The filter was retrieved directly by the wire-loop and organized thrombus and hyperplastic intima adhered to the filter.

of the guide wire above the filter helped to avoid snaring the filter struts and ensured that the wire-loop was able to snare the hook. Third, this technique can potentially be applied to drum-type filters as well as conical filters. When 
there was no interstice between the IVC wall and the filter, the homemade pigtail catheter was able to guide the wire to pass through the interstice of separate filter struts to form a wire-loop to stretch the filter hook away from the IVC wall. Subsequently, the conventional snare technique was performed easily. The long sheath may advance over the wire-loop to retrieve the filter directly if the wire-loop cannot stretch the filter hook away from the IVC wall because the filter hook and struts are embedded in the cava wall completely. Lastly, this technique can be performed using $11 \mathrm{~F}$ or $12 \mathrm{~F}$ long sheaths with less trauma.

In conclusion, the modified wire-loop snare technique could be performed easily to improve the retrieval rate of retrievable filters with severe tilt and embedded hooks. This technique could be used for the retrieval of two types of filters (OptEase and Celect). When CT angiography or intraoperative angiogram reveals severe tilt filter and embedded hooks, we recommend performing this wire-loop snare technique directly without trying to perform the routine snare technique.

\section{Footnotes}

Authors' Contributions: Yuewei Wang and Haofu Wang were responsible for the idea and initial manuscript of the article. Jun Li and Hai Xin collected and analyzed data. All authors approved the final version of the manuscript.

Conflict of Interests: All authors declare there is no conflict of interest.

Ethical Approval: Ethics Committee approval was obtained from the Institutional Ethics Committee of the Affiliated Hospital of Qingdao University. All patients approved to this study.

Financial Disclosure: All authors have no financial interests related to the material in the manuscript.

Funding/Support: No funding was received.

\section{References}

1. Angel LF, Tapson V, Galgon RE, Restrepo MI, Kaufman J. Systematic review of the use of retrievable inferior vena cava filters. J Vasc Interv Radiol.2011;22(11):1522-1530 e3. doi:10.1016/j.jvir.2011.08.024.[PubMed: 22024114].

2. McLoney ED, Krishnasamy VP, Castle JC, Yang X, Guy G. Complications of Celect, Gunther tulip, and Greenfield inferior vena cava filters on CT follow-up: A single-institution experience. J Vasc Interv Radiol. 2013;24(11):1723-9. doi: 10.1016/j.jvir.2013.07.023. [PubMed: 24041915].

3. Bikdeli B, Jimenez D, Kirtane AJ, Bracken MB, Spencer FA, Monreal $M$, et al. Systematic review of efficacy and safety of retrievable inferior vena caval filters. Thromb Res. 2018;165:79-82. doi: 10.1016/j.thromres.2018.03.014. [PubMed: 29579576]. [PubMed Central: PMC5943161].

4. Al-Hakim R, McWilliams JP, Derry W, Kee ST. The hangman technique: A modified loop snare technique for the retrieval of inferior vena cava filters with embedded hooks.JVasc Interv Radiol.2015;26(1):107-10. doi: 10.1016/j.jvir.2014.04.002. [PubMed: 25541448].

5. Foley PJ, Nathan DP, Wang GJ, Woo EY, Stavropoulos SW, ShlanskyGoldberg RD, et al. A "fall-back" technique for difficult inferior vena cava filter retrieval. J Vasc Surg. 2012;56(6):1629-33. doi: 10.1016/j.jvs.2012.02.063. [PubMed: 22607712].

6. Van Ha TG, Vinokur O, Lorenz J, Regalado S, Zangan S, Piano G, et al. Techniques used for difficult retrievals of the Gunther Tulip inferior vena cava filter: experience in 32 patients. J Vasc Interv Radiol. 2009;20(1):92-9. doi: 10.1016/j.jvir.2008.10.007. [PubMed:19026562].

7. Avery A, Stephens M, Redmond K, Harper J. Initial experience using the rigid forceps technique to remove wall-embedded IVC filters. J Med Imaging Radiat Oncol. 2015;59(3):306-11. doi: 10.1111/17549485.12299. [PubMed: 25754369].

8. Stavropoulos SW, Ge BH, Mondschein JI, Shlansky-Goldberg RD, Sudheendra D, Trerotola SO. Retrieval of tip-embedded inferior vena cava filters by using the endobronchial forceps technique: Experience at a single institution. Radiology. 2015;275(3):900-7. doi: 10.1148/radiol.14141420. [PubMed: 25581368].

9. Shukla PA, Nwoke F, Menon S, Halpert E. Dual access snare-over-wire method for retrieval of option inferior vena cava filters. J Vasc Interv Radiol.2014;25(10):1652-4. doi:10.1016/j.jvir.2014.06.014. [PubMed: 25255954].

10. Wang Y, Guan X, Wang H. A modified wire-loop snare technique for the retrieval of inferior vena cava filter with embedded hook. SAGE Open Med Case Rep. 2017;5:2050313X17745443. doi: 10.1177/2050313X17745443. [PubMed: 29238583]. [PubMed Central: PMC5721950]. 